\title{
Erratum to: Core outcome domains for clinical trials in non-specific low back pain
}

\author{
Alessandro Chiarotto ${ }^{1} \cdot$ Richard A. Deyo $^{2} \cdot$ Caroline B. Terwee $^{3} \cdot$ Maarten Boers $^{3}$ • \\ Rachelle Buchbinder ${ }^{4} \cdot$ Terry P. Corbin $^{5} \cdot$ Leonardo O. P. Costa $^{6,7} \cdot$ Nadine E. Foster $^{8}$. \\ Margreth Grotle $^{9}$ Bart W. Koes ${ }^{10}$ - Francisco M. Kovacs ${ }^{11}$ - Chung-Wei Christine Lin ${ }^{7}$. \\ Chris G. Maher ${ }^{7} \cdot$ Adam M. Pearson $^{12}$ - Wilco C. Peul ${ }^{13}$ - Mark L. Schoene ${ }^{14}$. \\ Dennis C. Turk ${ }^{15} \cdot$ Maurits W. van Tulder ${ }^{1}$ Raymond W. Ostelo ${ }^{1,3}$
}

Received: 29 April 2015/Revised: 29 April 2015/Accepted: 29 April 2015/Published online: 8 May 2015

(C) Springer-Verlag Berlin Heidelberg 2015

\section{Erratum to: Eur Spine J DOI 10.1007/s00586-015-3892-3}

The authors of the manuscript would like to add an acknowledgment to the Wetenschappelijk College Fysiotherapie (WCF) of the Royal Dutch Society for Physical Therapy (KNGF) that funded this study. The authors wish also to express that this funding body did not have any role in design, conduction, analysis, and interpretation of data, nor in writing this manuscript and deciding to submit this manuscript for publication. The views expressed in the manuscript are those of the authors and do not necessarily reflect those of their funding bodies.

The online version of the original article can be found under doi:10.1007/s00586-015-3892-3.

Alessandro Chiarotto

a.chiarotto@vu.nl

1 Department of Health Sciences, Faculty of Earth and Life Sciences, EMGO+ Institute for Health and Care Research, VU University Amsterdam, de Boelelaan 1085, room U-601, 1081 HV Amsterdam, The Netherlands

2 Departments of Family Medicine, Internal Medicine, Public Health and Preventive Medicine, Oregon Institute of Occupational Health Sciences, Oregon Health and Science University, Portland, USA

3 Department of Epidemiology and Biostatistics, EMGO+ Institute for Health and Care Research, VU University Medical Center, Amsterdam, The Netherlands

4 Monash Department of Clinical Epidemiology, Cabrini Institute and Department of Epidemiology and Preventive Medicine, School of Public Health and Preventive Medicine, Monash University, Melbourne, Australia

5 Cochrane Collaboration Back Review Group, Maple Grove, USA

6 Masters and Doctoral Programs in Physical Therapy, Universidade Cidade de Sao Paulo, Sao Paulo, Brazil

7 The George Institute for Global Health, Sydney Medical School, The University of Sydney, Sydney, Australia
8 Arthritis Research UK Primary Care Centre, Institute of Primary Care and Health Sciences, Keele University, Keele, UK

9 Oslo and Akershus University College of Applied Sciences, Faculty of Health Sciences and FORMI, Oslo University Hospital, Oslo, Norway

10 Department of General Practice, Erasmus MC, University Medical Center, Rotterdam, The Netherlands

11 Spanish Back Pain Research Network, Fundacion Kovacs, Palma de Mallorca, Spain

12 Department of Orthopaedic Surgery, Dartmouth-Hitchcock Medical Center, Lebanon, USA

13 Department of Neurosurgery, Leiden University Medical Center, Leiden, The Netherlands

14 Cochrane Collaboration Back Review Group, Newbury, USA

15 Department of Anesthesiology and Pain Medicine and Center for Pain Research on Impact, Measurement and Effectiveness, University of Washington, Seattle, USA 